University of Wollongong

Research Online

$1-1-2009$

\title{
Linkage, coordination and integration: Evidence from rural palliative care
}

Malcolm Masso

University of Wollongong,mmasso@uow.edu.au

Alan Owen

University of Wollongong, aowen@uow.edu.au

Follow this and additional works at: https://ro.uow.edu.au/ahsri

Research Online is the open access institutional repository for the University of Wollongong. For further information contact the UOW Library: research-pubs@uow.edu.au 


\title{
Linkage, coordination and integration: Evidence from rural palliative care
}

\author{
Abstract \\ OBJECTIVE: Review the findings from the evaluations of three rural palliative care programs. DESIGN: \\ Review by the authors of the original material from each evaluation. The conceptual framework for the \\ review was provided by the work of Leutz, including his distinction between linkage, coordination and full \\ integration. SETTING: Community-based palliative care in rural Australia. INTERVENTIONS: Fifteen \\ projects across all six states of Australia that focused on integration between general practitioners and \\ other community-based health providers. RESULTS: The projects set out to improve networking and \\ collaboration between providers; improve coordination and integration of care for patients; reduce \\ duplication of services; and achieve a multidisciplinary, collaborative approach to palliative care. The \\ most common interventions were establishment of formal governance structures, provision of education \\ programs, case conferencing, dissemination of information, development of formal arrangements, \\ development of protocols and use of common clinical assessment tools. The terms 'integration' and \\ 'coordination' were used frequently but without clear definitions. Coordination required someone \\ specifically designated to do the coordinating, usually a nurse. Formal arrangements to improve linkage \\ and coordination were difficult to maintain. The main mechanism to achieve full integration was the \\ development of common clinical information systems. CONCLUSIONS: The 'laws' proposed by Leutz and \\ the concepts of linkage, coordination and full integration provide a useful framework to understand the \\ barriers to integrating GPs and other health providers. It is important to be clear on what level of \\ integration is required. Improving links might be sufficient (and realistic), rather than striving for full \\ integration.
}

\section{Keywords}

coordination, linkage, care, palliative, rural, evidence, integration

\section{Publication Details}

Masso, M. \& Owen, A. (2009). Linkage, coordination and integration: Evidence from rural palliative care. Australian Journal of Rural Health, 17 (5), 263-267. 


\title{
Original Article
}

\section{Linkage, coordination and integration: Evidence from rural palliative care}

\author{
Malcolm Masso and Alan Owen \\ Centre for Health Service Development, University of Wollongong, Wollongong, New South Wales, \\ Australia
}

\begin{abstract}
Objective: Review the findings from the evaluations of three rural palliative care programs.

Design: Review by the authors of the original material from each evaluation. The conceptual framework for the review was provided by the work of Leutz, including his distinction between linkage, coordination and full integration.
\end{abstract}

Setting: Community-based palliative care in rural Australia.

Interventions: Fifteen projects across all six states of Australia that focused on integration between general practitioners and other community-based health providers.

Results: The projects set out to improve networking and collaboration between providers; improve coordination and integration of care for patients; reduce duplication of services; and achieve a multidisciplinary, collaborative approach to palliative care. The most common interventions were establishment of formal governance structures, provision of education programs, case conferencing, dissemination of information, development of formal arrangements, development of protocols and use of common clinical assessment tools. The terms 'integration' and 'coordination' were used frequently but without clear definitions. Coordination required someone specifically designated to do the coordinating, usually a nurse. Formal arrangements to improve linkage and coordination were difficult to maintain. The main mechanism to achieve full integration was the development of common clinical information systems.

Conclusions: The 'laws' proposed by Leutz and the concepts of linkage, coordination and full integration provide a useful framework to understand the barriers to integrating GPs and other health providers. It is

Correspondence: Malcolm Masso, Centre for Health Service Development, University of Wollongong, Wollongong, New South Wales, 2522, Australia. Email: mmasso@uow.edu.au

Accepted for publication 3 June 2009. important to be clear on what level of integration is required. Improving links might be sufficient (and realistic), rather than striving for full integration.

KEY WORDS: community-based service delivery, coordinated care, health program evaluation, primary health care, rural GP.

\section{Introduction}

The continuing concern for integrating the specialist and generalist components of primary health care has been internationally recognised for several decades ${ }^{1,2}$ and over the same period has been a recurring theme in Australian health reform commentaries. ${ }^{3-5}$

Integration in primary health care is of most value to patients with complex and chronic conditions. ${ }^{6}$ There are many references in the literature to integration, continuity, coordination, collaboration, partnerships and 'improving links', but little consensus on what these terms mean. It has been observed that almost every article on integration starts with the comment that 'there is no common definition'?

The most useful conceptualisation we have found (consistent with similar views described in a paper on case studies of New South Wales regional models ${ }^{6}$ ) is the work of Leutz who examined a range of 'integration efforts' and then proposed five laws of integration:

- You can integrate all of the services for some of the people, some of the services for all of the people, but you cannot integrate all of the services for all of the people

- Integration costs before it pays

- Your integration is my fragmentation

- You cannot integrate a square peg and a round hole

- The one who integrates calls the tune. ${ }^{8}$

In illustrating the first law, Leutz refers to three different levels of integration - linkage, coordination and full integration. Linkage refers to the relationships between systems that serve whole populations without relying on any special attention to the links, but rather a shared understanding of when, for example, to initiate a 
What is already known on this subject:

- Previously reported work on efforts to improve integration and coordination of care in rural areas has focused on single projects or single programs.

- There is little consensus in the literature on the definitions of integration, coordination and related terms.

- The evidence base for models of primary health care in rural and remote Australia is largely based on descriptive accounts rather than sound evaluations.
What this study adds:

- This study reflects on the results from three evaluations of rural palliative care programs using a particular organising framework.

- The study provides empirical testing of the laws of integration proposed by Leutz and builds on previous work using those laws.

- When setting out to improve integration, it is important to be clear on what level of integration is required in a given setting. Improved links might be sufficient rather than full integration. referral to another agency. Coordination by contrast requires structures and individuals with specific responsibility to 'coordinate', with the majority of the work undertaken by separate structures within existing systems. Full integration is more likely to occur when resources from multiple systems are pooled. ${ }^{8}$

The operational differences between the three levels are well illustrated by the flow of information. With linkage, information is provided when asked for and asked for when needed; coordination requires information sharing to be defined with reports provided routinely; full integration relies on a common record that is used as part of daily practice. ${ }^{8}$

Leutz's five laws and three levels of integration provided the conceptual framework for this paper, which focuses on integration between general practitioners and other community-based health providers. Integration was a feature of nine projects to improve palliative care in rural communities and a program that included a wider variety of projects but with a sub-set seeking to improve integration. General practitioners were involved in all the 'integration projects'. Our involvement in the evaluation of the 'integration projects' provided the catalyst for the paper.

\section{Methods}

Three evaluations of rural palliative care provided the data for analysis:

1. The evaluation of the Griffith Area Palliative Care Service, known more generally as the GAPS model..$^{9,10}$

2. The evaluation of the Caring Communities Program (CCP), which consisted of 37 projects addressing several themes, including: improve community awareness of palliative care, deliver education to health professionals, create effective partnerships between providers and coordinate care for dying people. $^{11}$
3. The evaluation of the Rural Palliative Care Program (RPCP), which consisted of eight projects, each implementing a model of care informed by the work in Griffith. ${ }^{12}$

Each evaluation was approved by the University of Wollongong Human Research Ethics Committee and used a consistent methodology of process and outcome measurement. ${ }^{13}$

The original documentation from each evaluation was reviewed to identify what each of the projects was trying to achieve, the most frequently used interventions and the lessons learnt, with a particular focus on the issue of coordination and integration. Relevant material from this process of review was entered into an Excel spreadsheet and sorted using the concept of matrix displays to identify commonalities between projects and key themes. ${ }^{14}$ Documentation included reports on the evaluation of each project, program evaluations for the CCP and RPCP and documents generated either by those undertaking the projects or the evaluations.

One author (M.M.) undertook the initial review, with secondary review by another author (A.O.). One or other of the authors was involved in each evaluation; hence much of the review was a process of reflection and re-framing of what was already known. The review synthesised the evidence of what the projects did and what they achieved.

\section{Results}

The three programs (GAPS, CCP and RPCP) represented a total of 46 projects devoted to improving palliative care. Of these, 15 projects (GAPS, six from the CCP and all eight from the RPCP) had a prominent role for GPs and formed the basis for this review of evidence.

Four projects were undertaken in Queensland, three in New South Wales, two in Victoria, one in South 
Australia, two in Tasmania and three in Western Australia. All 15 projects took place in rural areas with an emphasis on community-based services. In the case of 12 projects funding was allocated to divisions of general practice. Total funding was approximately $\$ 5$ million, with projects typically running over a period of 18 months to three years.

In their own terminology, the 15 projects aimed to do one or more of the following:

- Improve networking and collaboration between providers

- Achieve greater coordination between palliative care service providers

- Reduce duplication of services

- Improve integration of care for patients, families and carers

- Develop an integrated local service or palliative care system

- Ensure timely and coordinated care by GPs within a multidisciplinary team

- Achieve a multidisciplinary, collaborative approach to palliative care.

The most common interventions included the establishment of formal governance structures, provision of education programs, multidisciplinary team meetings involving GPs (including teleconferencing), dissemination of information (e.g. guidelines, service directories, newsletters), development of formal arrangements (e.g. memoranda of understanding), development of protocols and the use of common clinical assessment tools (Table 1).

TABLE 1: Interventions to improve coordination and integration

\begin{tabular}{ll}
\hline & No. of \\
Intervention & projects \\
\hline $\begin{array}{l}\text { Dissemination of information to GPs, including } \\
\text { resource kits and service directories }\end{array}$ & 13 \\
$\begin{array}{l}\text { Provision of education programs } \\
\text { Nurse coordinators to improve coordination of }\end{array}$ & 12 \\
$\quad$ patient care and links between providers & 11 \\
$\begin{array}{l}\text { Multidisciplinary team meetings with GP } \\
\text { involvement }\end{array}$ & 10 \\
$\begin{array}{l}\text { Use of common clinical assessment tools } \\
\text { Improved links between GPs and specialist }\end{array}$ & 8 \\
$\quad$ palliative care providers & 8 \\
Policies, protocols or procedures shared between & 8 \\
$\quad$ local heath providers & 7 \\
Use of patient-held record & 5 \\
Establishment of formal governance structures \\
$\begin{array}{l}\text { Memoranda of understanding between divisions of } \\
\quad \text { general practice and service providers }\end{array}$ & 4 \\
\hline
\end{tabular}

Multidisciplinary team meetings were typically used as a mechanism to bring health professionals together, but these meetings tended to focus on communication (linkage) rather than decision-making, care planning and review (coordination/integration). Various strategies were employed to involve GPs in these meetings, with differing degrees of success, and the meetings proved to be difficult to sustain.

The terms 'integration' and 'coordination' were used frequently by project teams, without any clear definition of what these terms meant within the context of each project. When projects did undertake what they saw as specific interventions to improve integration, this typically focused on improving links between providers rather than improving coordination and integration. Dissemination of information, by whatever means, was difficult to sustain. Coordination required someone specifically designated to do the coordinating, usually a nurse. Formal arrangements, such as memoranda of understanding or protocols, used to improve both linkage and coordination, were usually difficult to maintain.

The main mechanism to achieve the goal of full integration was the development of common clinical information systems. There was evidence in the early stages of the GAPS program that this did result in a 'common language' that facilitated communication between providers, but this again proved difficult to maintain. Each of the RPCP projects tried to use clinical assessment tools developed in the GAPS program as a 'common language', but in general achieved limited success. In part this was due to the difficulty in integrating an information system for one group of people (i.e. those requiring palliative care) within existing primary care information systems.

\section{Discussion}

Currently, there is not a good evidence base for models of primary health care in rural and remote Australia, primarily as a result of a reliance on descriptive accounts rather than sound evaluations. ${ }^{15}$ We have sought to add to the evidence base by reflecting on what was implemented and what was achieved by a series of 15 projects that sought to improve integration of palliative care in rural communities. Our findings are not necessarily representative of what would occur in all rural communities, but rather support the theoretical framework embodied in Leutz's five laws and his conceptualisation of integration. Our involvement in the evaluation of all the projects indicates the need for some caution regarding the findings, but we believe that this is mitigated by the common framework used to evaluate each project and the use of Leutz's work as an organising framework for this paper. 
Although each project was relatively small in scale, the interventions were quite complex, involving various interacting components seeking to change behaviour and practices, with adaptation to local circumstances. Evaluation of complex interventions is aided by a good theoretical understanding of how interventions cause change. ${ }^{16}$ Implementation of complex interventions should fit with the underlying theory or principles of the desired change, rather than trying to achieve standardisation across different settings. ${ }^{17}$

Part of the problem with attempts to 'integrate' providers is that structural and financial barriers exist at levels that local projects cannot easily influence. Linkage does not require confronting the funding and indemnity barriers to multidisciplinary teamwork, ${ }^{6}$ and GPs can use their knowledge of referral networks and services to link patients to other services. Systems can be put in place within general practice for reminders and follow-up processes that require little coordination. Much of this work reflects the notion that all integration is essentially local, which was not one of Leutz's original five laws, although he conceded in a later commentary that perhaps it should have been. ${ }^{18}$ Innovations adapted to the local context are more likely to be successfully implemented and become part of day-to-day practice. ${ }^{19}$

When combining the efforts of state-funded community health and social care services with Commonwealth primary medical care and private allied health providers, there are limits to the degree of integration that is possible. Vertical integration is more viable for specialist service provision for people with complex medical conditions. This was typically addressed by projects trying to improve links between local primary care providers and specialist palliative care providers, for example, the introduction of 'fly-in, fly-out' palliative care specialists or use of telephone consultations. More horizontal forms of integration face the challenge of fee for service GPs working with community-based services, which typically consist of individual health professionals providing services that are free at the point of delivery and supported by staff with responsibility for coordination.

GPs determine access to services based on medical need whereas other health care providers often determine access based on functional limitations where medical interventions are less relevant. This illustrates Leutz's point that you cannot integrate a square peg and a round hole and might explain in part the difficulty of having a GP division as a focus for integration efforts, as was found in a systematic review of system-wide models of comprehensive primary health care: 'There are limitations on the extent to which Divisions can further influence service delivery at a practice or local level, without significant change that involves the rest of the health system. In the absence of commissioning or significant enhancement of their contracting role, their success and effectiveness relies largely on their engagement with and responsiveness to their members' (McDonald et al., p. 57). ${ }^{20}$ Our review supports this finding.

One of Leutz's five laws ('your integration is my fragmentation') has particular resonance when considering the relationship between GPs and other health providers. The palliative care projects spent considerable time and effort trying to persuade busy providers (without the same motivation provided by project management) to 'integrate' by highlighting the special needs of palliative care clients and focusing on what services they require. For health care providers dealing with a broad spectrum of clients, the best example of which is GPs, coordination or integration for a sub-set of clients simply adds to the volume and complexity of their workload.

It might be that 'continuity' is just as important as integration. ${ }^{21}$ Continuity over time for an individual with chronic or life-limiting illness might not always require multiple providers, as was the case with the projects reported here where the vast majority of clinical work was provided by GPs and community nurses. Continuity between different providers does not always require a coordinator role, and continuity in information transfer does not always imply shared or sophisticated information systems. Improving continuity was primarily addressed by the projects we reviewed with activities to improve communication between providers, for example, in multidisciplinary team meetings where the focus was on communication about individual patients.

An important lesson is that when setting out to improve integration there should be clarity about what level of integration is required in a given setting. For example, when providing palliative care in rural settings, improving links might be sufficient (and realistic), rather than striving for full integration. As indicated by attempts to integrate clinical information systems, and as proposed by Leutz, integration in one part of a system results in fragmentation in another. This is essentially a planning and role delineation issue. For those with the most complex needs, continuity and coordination should be pursued, given the evidence of improved outcomes where more disciplines are involved. ${ }^{22}$

The concept of 'wicked problems' was originally proposed to describe ill-defined design and planning problems that were messy, circular and aggressive, in contrast to the relatively 'tame' problems of mathematics, chess or puzzle solving where relatively straightforward logic can get to a solution. ${ }^{23}$ Wicked problems can be resolved in part with clearer planning up front and more modest goals, in this case aiming short of full integration. In palliative care projects with a plethora of community programs with state and Commonwealth 
and non-government auspices, understanding Leutz's third law ('your integration is my fragmentation') can help adjust collective expectations. Limiting the aims, tailoring the goals of integration to what makes most sense in a local setting and understanding the barriers and differing roles will also help greatly.

\section{Author contributions}

M.M., 60\%; A.O., 40\%.

\section{References}

1 Starfield B. The future of primary care: refocusing the system. New England Journal of Medicine 2008; 359: 2087, 2091.

2 Starfield B, Shi L, Macinko J. Contribution of primary care to health systems and health. Milbank Quarterly 2005; 83: 457-502.

3 National Health Strategy. Issues paper No. 1, The Australian Health Jigsaw: integration of health care delivery. National Health Strategy, 1991.

4 National Hospitals and Health Services Commission. A Community Health Program for Australia. Canberra: Australian Government Publishing Service, 1973.

5 Powell Davies G, Williams AM, Larsen K, Perkins D, Roland M, Harris MF. Coordinating primary health care: an analysis of the outcomes of a systematic review. Medical Journal of Australia 2008 (Apr 21); 188: S65-S68.

6 May J, Cooper R, Magin P, Critchley A. Integrated models or mayhem? Lessons learnt from three integrated primary health care entities in regional NSW. Australian Health Review 2008; 32: 595-603.

7 Fooks C. An integration journey: road trips from afar. The Change Foundation 2008. [Cited 20 April 2009]. Available from URL: http://www.changefoundation.ca/docs/Jan.25OHA.ppt\#262,1

8 Leutz WN. Five laws for integrating medical and social services: lessons from the United States and the United Kingdom. Milbank Quarterly 1999; 77: 77-110.

9 Eagar K, Owen A, Masso M, Quinsey K. The Griffith Area Palliative Care Service (GAPS): an evaluation of an Australian rural palliative care model. Progress in Palliative Care 2006; 14: 112-119.

10 Masso M, Fildes D, Quinsey K, Matete S. GAPS revisited: follow up evaluation of an Australian rural palliative care service. Progress in Palliative Care 2007; 15: 233-239.

11 Quinsey K, Williams K, Fildes D et al. Caring Communities: Evaluation of a National Palliative Care Program.
Wollongong: Centre for Health Service Development, University of Wollongong, 2006.

12 Quinsey K, Masso M, Fildes D et al. Providing Palliative Care in Rural Australia: results of a national program evaluation. Wollongong: Centre for Health Service Development, University of Wollongong, 2007.

13 Eagar K, Cranny C, Fildes D. Evaluation and Palliative Care: A Guide to the Evaluation of Palliative Care Services and Programs. Wollongong: Centre for Health Service Development, University of Wollongong, 2004.

14 Miles MB, Huberman AM. Qualitative Data Analysis: An Expanded Sourcebook, 2nd edn. Thousand Oaks, CA: Sage Publications, 1994.

15 Wakerman J, Humphreys J, Wells R, Kuipers P, Entwistle P, Jones J. Systematic Review of Primary Health Care Delivery Models in Rural and Remote Australia 19932006. Canberra: Centre for Remote Health - Flinders University \& Charles Darwin University, Monash University Faculty of Medicine, Nursing and Health Sciences, University of Queensland, 2006.

16 Craig P, Dieppe P, Macintyre S, Mitchie S, Nazareth I, Petticrew M. Developing and evaluating complex interventions: the new Medical Research Council guidance. British Medical Journal 2008; 337: 979-983.

17 Hawe P, Shiell A, Riley T. Complex interventions: how 'out of control' can a randomised controlled trial be? British Medical Journal 2004; 328: 1561-1563.

18 Leutz W. Reflections on integrating medical and social care: five laws revisited. Journal of Integrated Care 2005; 13: 3-12.

19 Greenhalgh T, Robert G, Macfarlane F, Bate P, Kyriakidou O. Diffusion of innovations in service organizations: systematic review and recommendations. Milbank Quarterly 2004; 82: 581-629.

20 McDonald J, Cumming J, Harris MF, Powell Davies G, Burns P. Systematic Review of System-Wide Models of Comprehensive Primary Health Care. Sydney: Research Centre for Primary Health Care and Equity, School of Public Health and Community Medicine, University of New South Wales, 2006.

21 Haggerty JL, Reid RJ, Freeman GK, Starfield BH, Adair CE, McKendry R. Continuity of care: a multidisciplinary review. British Medical Journal 2003; 327: 12191221.

22 Tieman J, Mitchell G, Shelby-James T et al. Integration, Coordination and Multidisciplinary Approaches in Primary Care: A Systematic Investigation of the Literature. Adelaide: Flinders University Department of Palliative and Supportive Services, 2006.

23 Rittel H, Webber M. Dilemmas in a general theory of planning. Policy Sciences 1973; 4: 155-169. 